\title{
NONLINEAR OBLIQUE INTERACTION OF LARGE AMPLITUDE INTERNAL SOLITARY WAVES
}

\author{
Keisuke Nakayama ${ }^{1}$, Taro Kakinuma ${ }^{2}$, Hidekazu Tsuji ${ }^{3}$ and Masayuki Oikawa ${ }^{4}$
}

\begin{abstract}
Solitary waves are typical nonlinear long waves in the ocean. The two-dimensional interaction of solitary waves has been shown to be essentially different from the one-dimensional case and can be related to generation of large amplitude waves (including 'freak waves'). Concerning surface-water waves, Miles (1977) theoretically analyzed interaction of three solitary waves, which is called "resonant interaction" because of the relation among parameters of each wave. Weakly-nonlinear numerical study (Funakoshi, 1980) and fully-nonlinear one (Tanaka, 1993) both clarified the formation of large amplitude wave due to the interaction ("stem" wave) at the wall and its dependency of incident angle. For the case of internal waves, analyses using weakly nonlinear model equations (e.g. Tsuji and Oikawa, 2006) suggest also qualitatively similar results. Therefore, the aim of this study is to investigate the strongly nonlinear interaction of internal solitary waves; especially whether the resonant behavior is found or not. As a result, it is found that the amplified internal wave amplitude becomes about three times as much as the original amplitude. In contrast, a "stem" is not found to occur when the incident wave angle is more than the critical angle, which has been demonstrated in the previous studies.
\end{abstract}

Keywords: variational principle; internal wave; two-layer system; soliton resonance; fully nonlinear; strong dispersive

\section{Introduction}

Solitary waves are typical nonlinear long waves in the ocean. The two-dimensional interaction of solitary waves has been shown to be essentially different from the one-dimensional case, and can be related to generation of large amplitude waves (including 'freak waves'). Concerning surface-water waves, Miles (1977) theoretically analyzed the interaction of three solitary waves, which can be called "resonant interaction" because of the relation among parameters of each wave, and applied the result to the oblique reflection of solitary waves at a wall. A weakly-nonlinear numerical study (Funakoshi, 1980) and a fully-nonlinear study (Tanaka, 1993) both clarified the formation of large amplitude waves due to the interaction ("stem" wave) at the wall and its dependency on the incident angle. For the case of internal waves, analyses using weakly nonlinear model equations (e.g. Tsuji and Oikawa, 2006) also suggested qualitatively similar results. However, as these solutions were obtained using weakly nonlinear model equations, Strongly nonlinear wave effects were not explored but were expected to change the amplification rate of the solitary waves.

Similar to surface waves, large amplitude internal waves occur in a stratified flow field which can induce resuspension and transport mass (Wallace and Wilkinson, 1988; Helfrich, 1992; Pierson and Weyhenmeyer, 1994; Antenucci and Imberger, 2001; Boegram et al., 2005; Boegman and Ivey, 2009; Nakayama and Imberger, 2010; Aghsaee et al., 2010; Nakayama et al., 2012). Nakayama and Imberger (2010) and Nakayama et al. (2012) demonstrated that long-term transport occurs due to intrusion under the density interface resulting from the breaking of internal waves over a uniform slope. Therefore, it is necessary to analyze large amplitude internal waves in the context of their capacity to induce long-term mass transport.

Choi and Camassa (1999) and Horn et al. (2001) show how to deal with one-dimensional internal solitary waves, but do not provide theoretical solutions of an internal solitary wave as an initial condition. Thus, we applied third-order internal solitary wave equations based on the ninth-order solitary wave equations (Mirie and Pennel, 1989) as an initial condition by using a fully nonlinear and strongly dispersive internal wave model (FSI model: Kakinuma, 2001; Nakayama and Kakinuma, 2010). The FSI equations were derived based on the variational principle, which was a modified version of the theory proposed by Luke (1967). The aim of this study was to investigate the strongly nonlinear interaction of internal solitary waves; especially whether resonant behavior was evident.

\footnotetext{
${ }^{1}$ Civil and Environmental Engineering, Kitami Institute of Technology, 165 Koen-cho, Kitami City, 090-8507, Japan

${ }^{2}$ Natural Science, Kagoshima University, 1-21-40 Korimoto, Kagoshima City, 890-0065, Japan

${ }^{3}$ Research Institute for Applied Mechanics, Kyushu University, 6-1 Kasuga-koen Kasuga City, 816-8580, Japan

${ }^{4}$ Intelligent Mechanical Engineering, Fukuoka Institute of Technology, 3-30-1 Wajiro-higashi Higashiku Fukuoka City, 811-0295, Japan
} 


\section{FSI model}

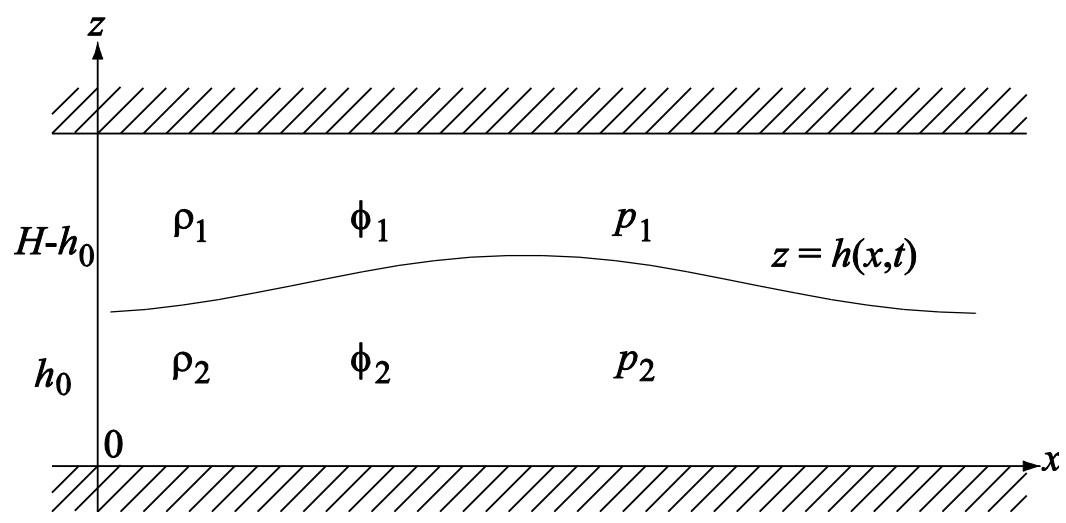

Figure 1. Schematic diagram of a two-layer system used in the FSI model.

Wave equations in a two-layer system with an irrotational flow field (Fig. 1) were obtained by expanding velocity potential using vertical profile functions.

$$
\begin{gathered}
\mathbf{u}_{i}=\nabla \phi_{i} \text { and } w_{i}=\partial \phi_{i} / \partial z \\
\phi_{i}(x, z, t)=\sum_{\alpha=0}^{N-1} Z_{i, \alpha}\left\{z, h_{i}(x)\right\} f_{i, \alpha}(x, t) \equiv Z_{i, \alpha} f_{i, \alpha}
\end{gathered}
$$

where $\rho_{1}$ and $\rho_{2}$ indicate the density in the upper and lower layers, $H$ and $h_{0}$ indicate the total and lower layer thickness, $\zeta$ is the interfacial displacement, $p_{1}$ and $p_{2}$ indicate the pressure in the upper and lower layer, $\nabla$ is a partial differential operator in the horizontal plane, i.e., $\nabla=(\partial / \partial x, \partial / \partial y), N$ is the number of vertically distributed functions and the sum rule of product is adopted for subscript $\alpha, f_{i, a}$ is the weight for the $i^{\text {th }}$-layer.

The definition of the vertical profile function as in Eq. 3 yields upper and lower layer equations.

$$
Z_{i, \alpha}=Z^{\alpha}
$$

Upper layer equations:

$$
\begin{gathered}
\eta^{a} \frac{\partial \eta}{\partial t}+\frac{1}{\alpha+\beta+1} \nabla\left(\eta^{\alpha+\beta+1} \nabla f_{1, \beta}\right)-\frac{\alpha \beta}{\alpha+\beta-1} \eta^{\alpha+\beta-1} f_{1, \beta}=0 \\
\eta^{\beta} \frac{\partial f_{1, \beta}}{\partial t}+\frac{1}{2} \eta^{\beta+\gamma} \nabla f_{1, \beta} \nabla f_{1, \gamma}+\frac{\beta \gamma}{2} \eta^{\beta+\gamma-2} f_{1, \beta} f_{1, \gamma}+g \eta+\frac{p_{1}}{\rho_{1}}=0
\end{gathered}
$$

Lower layer equations:

$$
\begin{gathered}
\eta^{a} \frac{\partial \eta}{\partial t}+\frac{1}{\alpha+\beta+1} \nabla\left\{\left(\eta^{\alpha+\beta+1}-b^{\alpha+\beta+1}\right) \nabla f_{2, \beta}\right\} \\
-\frac{\alpha \beta}{\alpha+\beta-1}\left(\eta^{\alpha+\beta-1}-b^{\alpha+\beta-1}\right) f_{2, \beta}=0 \\
\eta^{\beta} \frac{\partial f_{2, \beta}}{\partial t}+\frac{1}{2} \eta^{\beta+\gamma} \nabla f_{2, \beta} \nabla f_{2, \gamma} \\
+\frac{\beta \gamma}{2} \eta^{\beta+\gamma-2} f_{2, \beta} f_{2, \gamma}+g \eta+\frac{p_{1}+\left(\rho_{2}-\rho_{1}\right) g h_{1}}{\rho_{2}}=0
\end{gathered}
$$

In the FSI equations, the velocity potential is defined using expansion of the power function, which means that the higher the number of $N$, the higher the accuracy of the computation. When $N=1$, the FSI equations are the same as the shallow water equations. It should be noted that it is possible to apply the equations to surface waves by taking the upper and lower layer as air and water, respectively.

\section{3rd order internal solitary wave solution}

The shape of the internal solitary wave was obtained from Eqs. 8 to 16. 


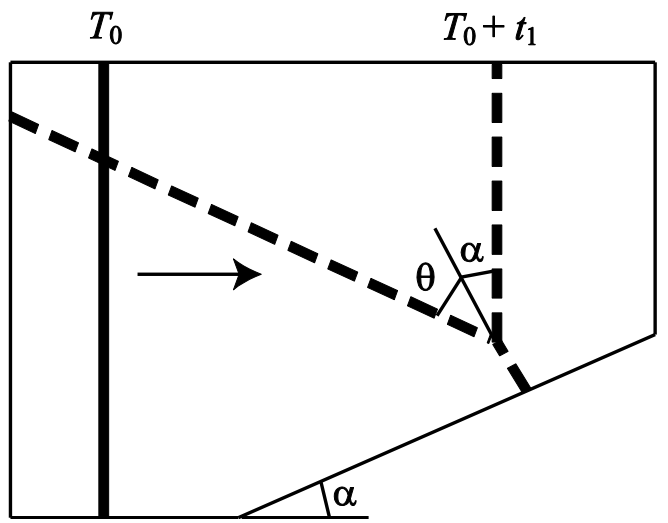

Figure 2. Computational domain.

Table 1 Incident angle and its amplitude for case 1 to case 8

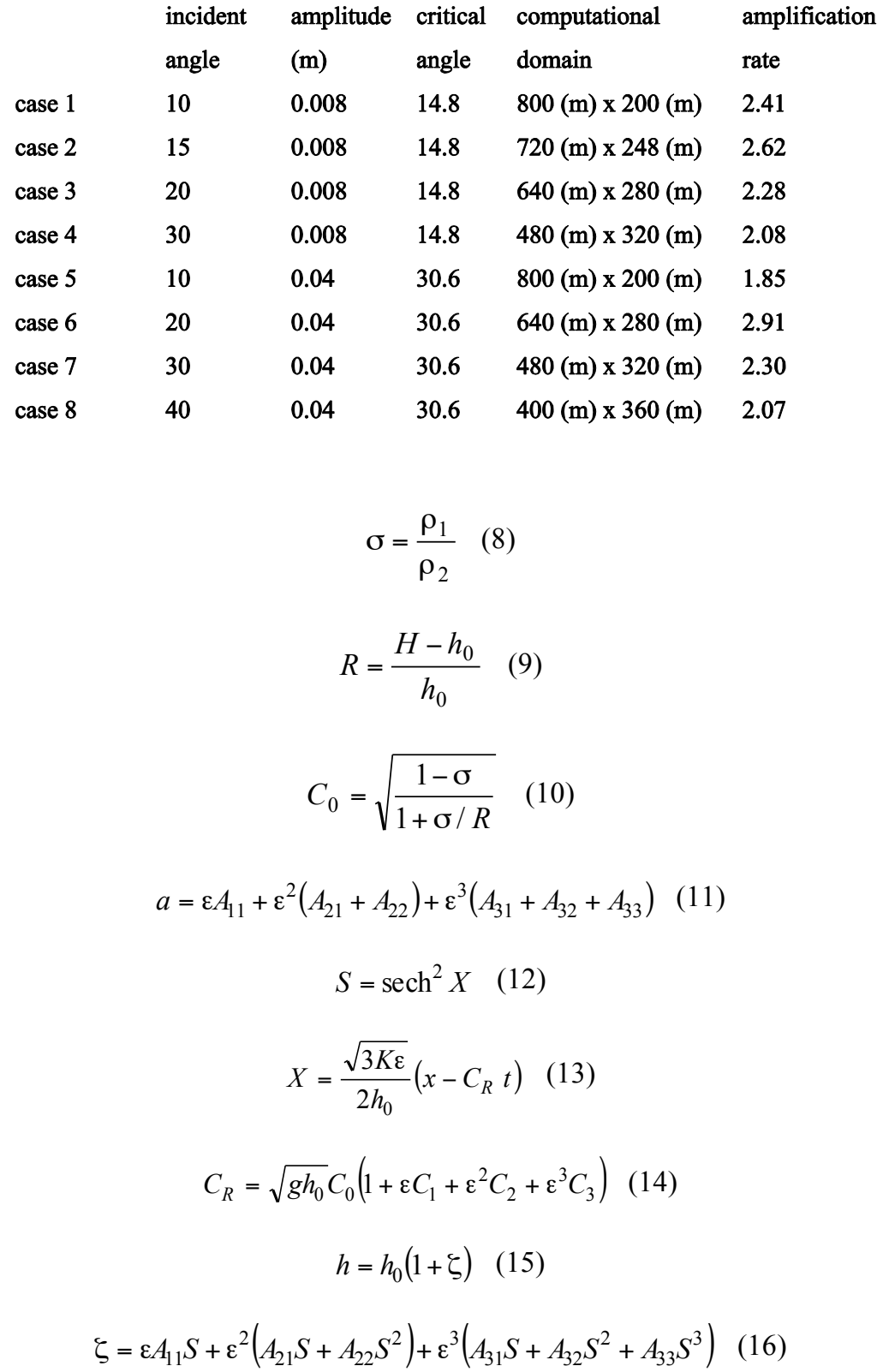




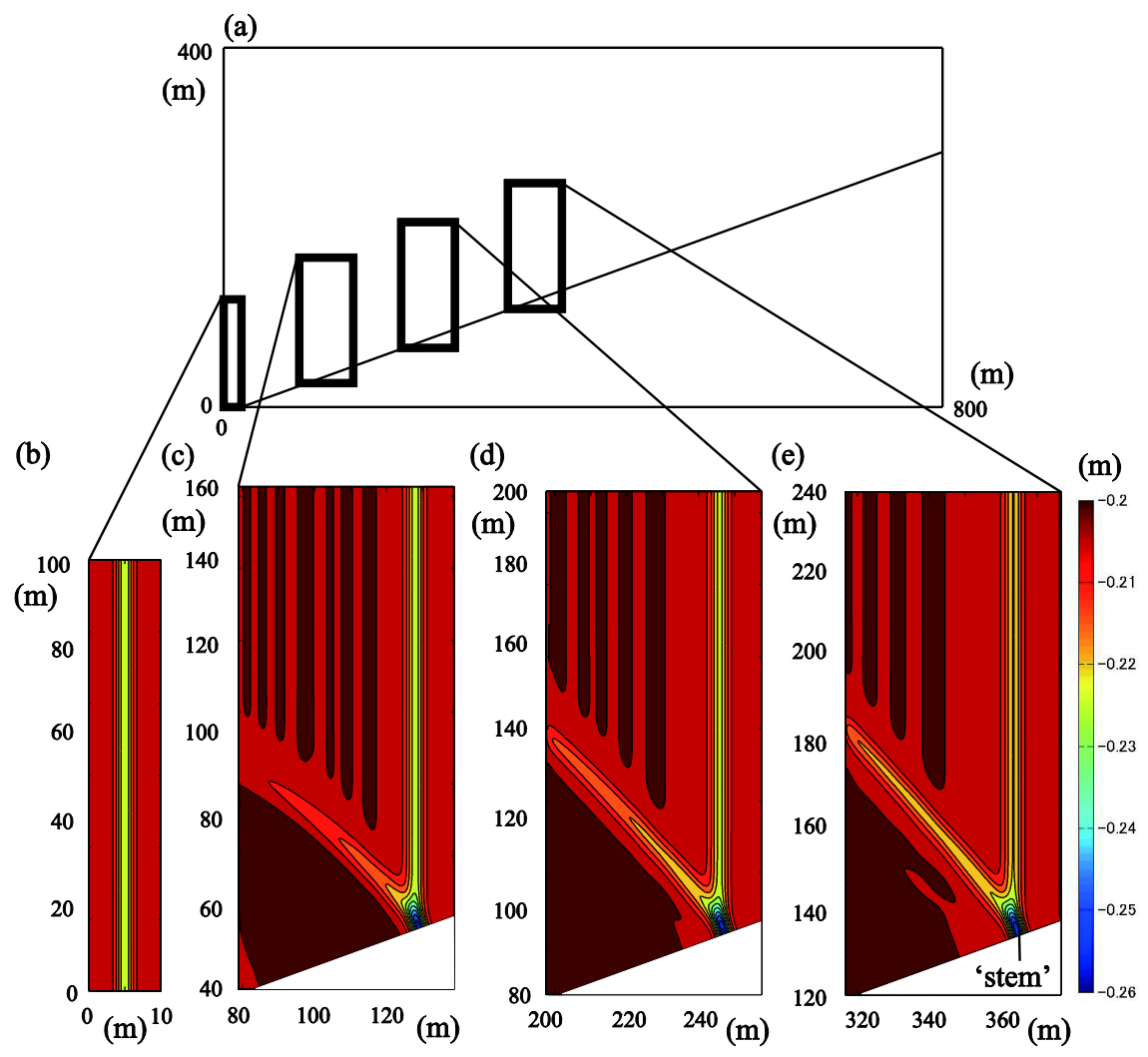

Figure 3. Interaction of internal solitary wave when the incident wave angle is 20 degrees. (a) Computational domain, $800 \mathrm{~m} \times 400 \mathrm{~m}$. (b) Initial condition. (c) $100 \mathrm{~s}$. (d) $200 \mathrm{~s}$. (e) $300 \mathrm{~s}$.

where $a$ is the amplitude of internal solitary wave, $h$ is the interfacial level, $K$ is the coefficient regarding spatial scale, $A_{i j}(i, j=1 \sim 3)$ indicates the coefficient for interfacial displacement and $C_{i}(i=$ $1 \sim 3)$ indicates the coefficient of wave speed.

\section{Computational domain and results}

To compute the resonance of solitary waves, we applied oblique boundary conditions for an incident solitary wave in the computational domain, as proposed by Funakoshi (1980) (Fig. 2). The upper and lower layer depths were $0.2 \mathrm{~m}$ and $0.8 \mathrm{~m}$, respectively, the specific density difference 2.0, and the amplitude given as $0.024 \mathrm{~m}$. Computations were conducted for 8 cases, in which the incident wave angle ranged from 15 to 40 degrees (Table 1). To save computational time, the actual computational region was limited to the 150 meshes around the incident internal solitary wave, which corresponds to $60 \mathrm{~m}$ in the $x$ coordinate. Elsewhere, a sponge layer was applied to reduce the reflection from the outside of the actual computational region. Parallel computing was applied using 8 CPUs.

Kadomtsev-Petviashvili (KP) theory can provide critical angle of oblique internal solitary waves in a two-dimensional domain shown as Eqs. 17 to 20 .

$$
\begin{aligned}
\sigma & =\frac{\rho_{1}}{\rho_{2}}(17) \\
\gamma & =\frac{h_{2}}{h_{1}} \\
p & =\frac{\sigma-\gamma^{2}}{\gamma(\gamma+\sigma)} \\
\tan \varphi & =\sqrt{3 p \frac{a}{h_{1}}}
\end{aligned}
$$


where $\varphi$ is the critical angle.

(a)

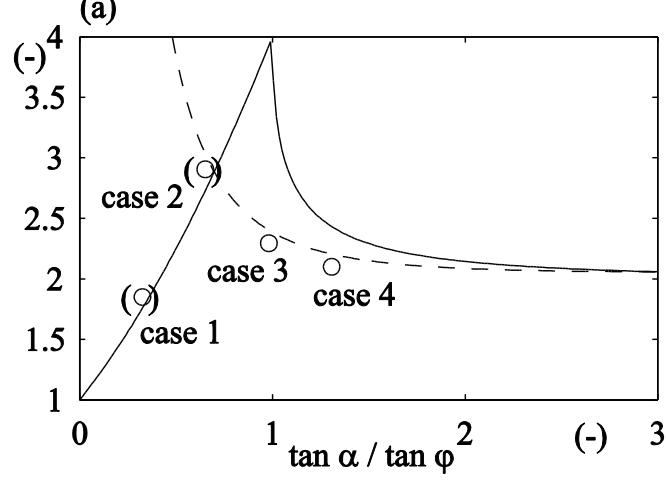

(b)

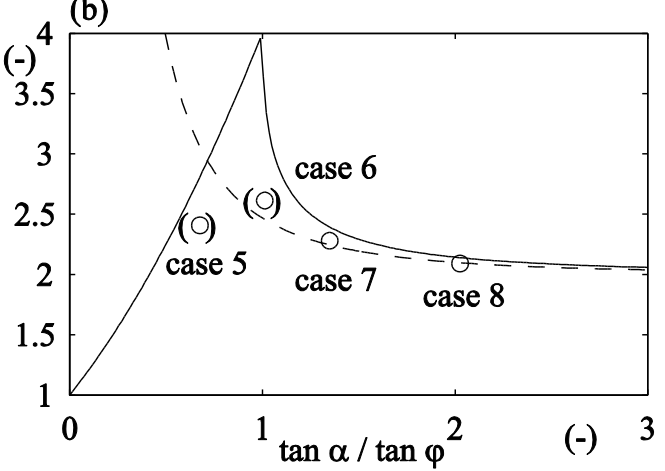

Figure 4. Amplification rate from computational results and theory. Circles indicate computational results, solid lines indicate the result of the KP theory, and broken lines indicate the solution based on the Boussinesq-type equations.

A "“stem"' was found to occur when the incident wave angle was less than the critical angle, and the "“stem"" length was confirmed to decrease with increasing incident wave angle. As a result, the amplified internal wave amplitude was found to be about three times as much as the original amplitude (Fig. 3). In contrast, a "'stem"" was not found to occur when the incident wave angle was more than the critical angle, which has been demonstrated in the previous studies.

\section{Discussion}

Amplification rate was compared with that of the KP theory and the solution based on Boussinesqtupe equations (Kakinuma and Nakayama, 2007; Nakayama K. and J. Imberger. 2010). It was found from the KP theory that Mach reflection was observed when the incident angle was smaller than the critical angle, and regular grazing reflection was found when the incident angle was larger than the critical angle (Eqs. 21 to 22).

$$
\begin{gathered}
\beta=\left(1+\frac{\tan \alpha}{\tan \varphi}\right)^{2} \text { for } \tan \alpha \leq \tan \varphi \\
\beta=4 /\left(1+\sqrt{1-\frac{\tan ^{2} \varphi}{\tan ^{2} \alpha}}\right) \text { for } \tan \alpha>\tan \varphi(22)
\end{gathered}
$$

where $\beta$ is amplification rate.

On the other hand, the theory based on the Boussinesq-type equations can provide regular nongrazing reflection shown as Eq. 23.

$$
\beta=2+p \frac{a_{0}}{h_{1}}\left(\frac{3}{2 \sin ^{2} \alpha}-3+2 \sin ^{2} \alpha\right)
$$

The maximum amplification rate for the 8 cases was found to be about 3 , which agreed with the results demonstrated by Tanaka (1993) and Li et al. (2011) (Fig. 4). Interestingly, the amplification rates for the cases excluding case 1 and case 5 showed that regular non-grazing reflection agreed with the computational results, which may indicate the limitation of using the KP theory (Kodama, 2010).

\section{REFERENCES}

Aghsaee P., L. Boegman and K. G. Lamb. 2010. Breaking of shoaling internal solitary waves, Journal of Fluid Mechanics, 659, 289-317. doi:10.1017/S002211201000248X

Antenucci, J..P. and J. Imberger. 2001. Energetics of long internal gravity waves in large lakes, Limnology and Oceanography, 46, 1760-1773.

Boegman, L., G. N. Ivey and J. Imberger 2005. The degeneration of internal waves in lakes with sloping topography, Limnology and Oceanography, 50, 1620-1637.

Boegman, L. and G. N. Ivey. 2009. Flow separation and resuspension beneath shoaling nonlinear internal waves, Journal of Geophysical Research, 114, C02018.

Choi, W. and R. Camassa. 1999. Fully nonlinear internal waves in a two-fluid system, Journal of Fluid Mechanics, 396, 1-36. 
Funakoshi, M. 1980. Reflection of obliquely incident solitary waves, Journal of the Physical Society of Japan, 49(6), 2371-2379.

Helfrich, K.R. 1992. Internal solitary wave breaking and run-up on a uniform slope, Journal of Fluid Mechanics, 243, 133-154.

Horn, D.A., J. Imberger and G.N. Ivey. 2001. The degeneration of large-scale interfacial gravity waves in lakes, Journal of Fluid Mechanics, 434, 181-207.

Kakinuma, T. 2001. A set of fully nonlinear equations for surface and internal gravity waves, Proceedings of 5th International Conference on Computer Modelling of Seas and Coastal Regions, WIT Press, 225-234.

Kakinuma T. and K. Nakayama. 2007. Numerical simulation of internal waves using a set of fully nonlinear internal wave equations, Annual Journal of Hydraulic Engineering, JSCE, 51, 169-174.

Kodama, Y. 2010. KP solitons in shallow water, Journal of Physics A: Mathematical and Theoretical, 43, 434004-434057. doi:10.1088/1751-8113/43/43/434004

Li, W., H. Yeh and Y. Kodama. 2011. On the Mach reflection of a solitary wave: revisited, Journal of Fluid Mechanics, 672, 326-357.

Luke, J.C. 1967. A variational principle for a fluid with a free surface, Journal of Fluid Mechanics, 27, 395-397. doi:10.1017/S0022112067000412.

Miles, J.W. 1977. Resonantly interacting solitary waves. Journal of Fluid Mechanics, 79, 171-179.

Mirie, R.M. and S.A. Pennell. 1989. Internal solitary waves in a two-fluid system, Physics of Fluids, A1(6), pp.986-991.

Mirie, R.M. and S.A. Pennell. 1989. Internal solitary waves in a two-fluid system, Physics of Fluids, A1(6), 986-991.

Nakayama K. and J. Imberger. 2010. Residual circulation due to internal waves shoaling on a slope, Limnology and Oceanography, 55, 1009-1023.

Nakayama K. and T. Kakinuma. 2010. Internal waves in a two-layer system using fully nonlinear internal-wave equations, International Journal for Numerical Methods in Fluids, 62(5), 574-590. doi: 10.1002/fld.2037

Nakayama K., T. Shintani, K. Kokubo, Y. Maruya, T. Kakinuma, K. Komai and T. Okada. 2012. Residual current over a uniform slope due to breaking of internal waves in a two-layer system, Journal of Geophysical Research, accepted.

Pierson, D.C. and G. A. Weyhenmeyer. 1994. High resolution measurements of sediment resuspension above an accumulation bottom in a stratified lake, Hydrobiologia, 284, 43-57.

Tanaka, M. 1993. Mach reflection of a large-amplitude solitary wave, Journal of Fluid Mechanics, 248, 637-661. doi: http://dx.doi.org/10.1017/S0022112093000941

Tsuji, H. and M. Oikawa. 2007. Oblique interaction of solitons in an extended Kadomtsev-Petviashvili equation, Journal of Physical Society of Japan, 76, 84401-84408.

Wallace, B.C., and D. L. Wilkinson. 1988. Run-up of internal waves on a gentle slope in a two-layered system, Journal of Fluid Mechanics, 191, 419-442. 